\title{
Incubation time of relapses after treatment of paucibacillary leprosy
}

\author{
S R PATTYN Co-ordinator, Collaborative Study Group* for \\ the Treatment of Leprosy in Zaire, Burundi and Rwanda \\ Institute for Tropical Medicine, Nationalestraat 155, B-2000 \\ Antwerp, Belgium
}

Accepted for publication 18 August 1983

\begin{abstract}
Summary Data are presented on the incubation time of 21 relapses af ter stopping dapsone monotherapy in paucibacillary leprosy in Central Af rica. The results are comparable with those of other studies: $50 \%$ of relapses occur during the first $2-3$ years. This figure is most important to analyse the results of drug trials in paucibacillary leprosy. This figure should also be relevant for regimens including more bactericidal drugs than dapsone, since the kind of antibacterial treatment should influence the minimal necessary duration of treatment but not the incubation time of relapses.
\end{abstract}

The same mechanisms prevailing in relapses of multibacillary leprosy, their incubation periods should be identical.

The two most important criteria for the evaluation of treatment regimens in infectious diseases are improvement or disappearance of symptoms, as evaluated by clinical, bacteriological, histopathological and other procedures, and incidence of relapses. For the organization of studies of treatment regimens it is most important to know the length of the incubation time of relapses, since this should determine the duration of the follow-up period after treatment has ceased. In studies on the treatment of pulmonary tuberculosis, originally, follow-up periods of 2 years were chosen, but later reduced to 1 year, when it became apparent that most relapses, after stopping treatment, appeared during the first year.

Now that similar treatment trials are envisaged in leprosy, it is equally important to gather information on incubation times of relapses in leprosy. We

* Members of the Collaborative Study Group. Zaire: N Bossaer, L Breugelmans, L Coussens, H Cuyckens, J Deverchin, F Douchy, G Groenen, L Janssens, N M Makela, E Nollet, R Petit, A M Passagez, B Rosano, J Van Bockstaele, J Verlinden, R Wattelet; Burundi: J Bourland, L Desmet; Rwanda: M Boen, E Schacht, M Valette; Antwerp: G Hooft, S R Pattyn, A Van Aerde, G Van Loo. 
present here the results of our Study Group concerning the incubation time of relapses during the last 2 years in Central Africa: Zaire, Rwanda and Burundi, and compare them with similar data from the literature.

\section{Materials and methods}

In the whole of Rwanda and Burundi, and in parts of Zaire, Collaborating Centres are based in rural hospitals and visit peripheral dispensaries and health centres at intervals of 1, 2, 6 or 12 months. Patient detection is passive.

For each patient not under treatment, a case history is taken, and clinical and bacteriological examinations are performed and recorded on standard clinical files, copies of which are forwarded to the Leprosy Laboratory, Institute for Tropical Medicine, Antwerp, together with a skin biopsy if the patient has a paucibacillary (PB) form of the disease (paucibacillary leprosy being characterized by a bacterial index of less than two at any of at least three skin smears taken either from one earlobe and two skin lesions or two earlobes and one skin lesion).

The data are analysed in Antwerp. A relapse is defined as the appearance of new skin lesions, confirmed as leprosy through either bacteriology or histopathology, in a patient for whom clinical files are available documenting the previous diagnosis, and considered cured by a medical doctor or an experienced

Table 1. Incubation time for relapses after treatment of paucibacillary leprosy

\begin{tabular}{ll} 
Incubation time* & No. \\
\hline $2 / 12$ & 1 \\
$6 / 12$ & 1 \\
$9 / 12$ & 2 \\
1 & 3 \\
$14 / 12$ & 1 \\
2 & 2 \\
3 & 3 \\
4 & 1 \\
5 & 2 \\
6 & 1 \\
8 & 1 \\
20 and more & 3 \\
\hline
\end{tabular}

thereof.

* In years or fractions 
paramedical worker who had deliberately taken the decision to stop treatment. In French-speaking African countries the expression '(E)OST (en) observation sans traitement' (under observation without treatment) is currently used. Thus were excluded, patients who presented themselves after absconding and claiming they had new lesions, and patients who claimed they had been cured in the past but for whom no clinical files, testifying the anamnestic data were available.

In the present study relapses are not related to a well-defined population of previously treated patients but were noted as they presented spontaneously. All patients had been treated with dapsone monotherapy mostly in increasing dosages at the start, and maintained on dapsone 50 or $100 \mathrm{mg}$ daily, in self-administration.

\section{Results}

A total of 21 paucibacillary relapses were documented.

The incubation times (Table 1) varied from 2 months to 20 years and more, with a mean of 5 years, Figure 1. Based on an underlying exponential distribution of the relapse times, the median was calculated as $3 \frac{1}{2}$ years. The $95 \%$ confidence intervals, based on the variance calculation by Greenwood's formula and a

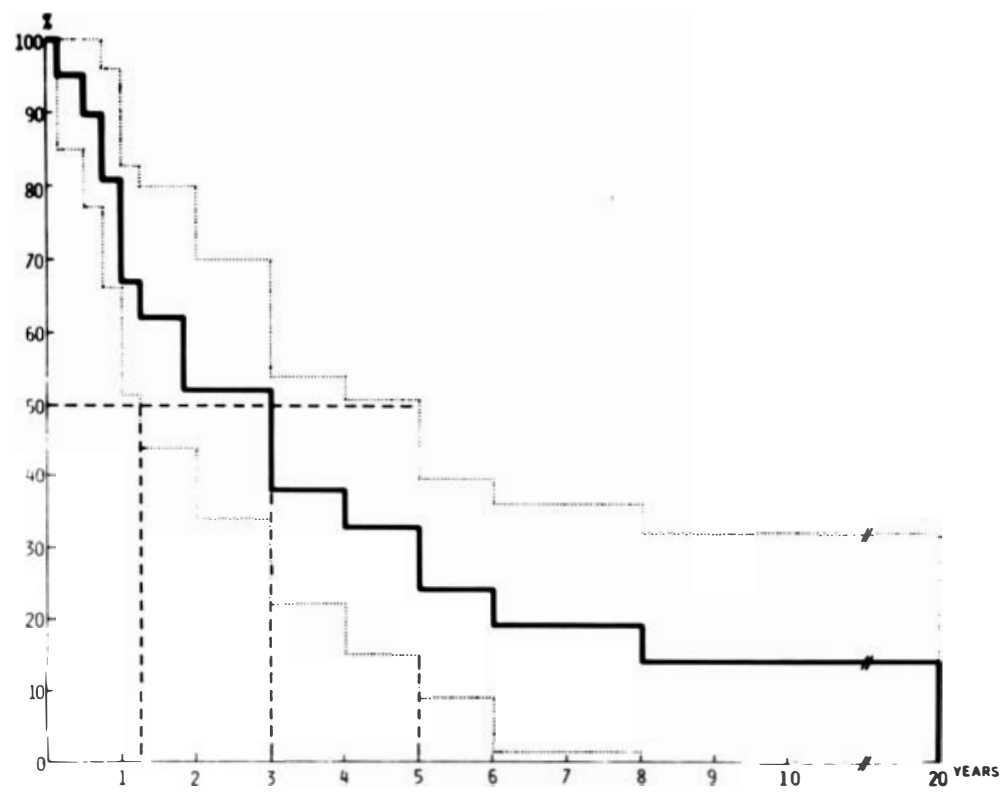

Figure 1. Incubation time for relapses with $95 \%$ confidence intervals. - , distribution of the relapse incubation times; --.---, $95 \%$ confidence intervals of the distribution of the relapse incubation times. 
bilateral hypothesis, are represented graphically in Figure 1, and are situated between $1 \frac{1}{4}$ and 5 years.

There was no correlation between the duration of previous, unsupervised, dapsone therapy and the incubation time for relapse. The minimum duration of therapy was 2 years ( 1 case) but most patients had been treated for 5 years or more, but since treatment was not supervised the actual intake of drug is unknown.

All cases had originally been diagnosed and classified as PB leprosy. At relapse, 17 were classified histologically as BT and 4 as TT leprosy, the incubation times for relapse in the latter were 2 and 5 months and 2 and 5 years respectively.

\section{Discussion}

For the evaluation of the efficacy of treatment regimens in leprosy, figures are urgently needed on the incubation time of relapses after stopping treatment, to allow the determination of the minimal necessary duration of follow-up. There are not so many data on this subject in the literature and most are concerned with multibacillary leprosy, whereas for PB leprosy the aim of most studies was to define the length of dapsone treatment necessary to prevent relapses.

In 1954, Lowe' mentions that in a group of patients followed for periods from a few weeks to 4 years - on average 22 months -8 relapses were observed. Seven occurred between 3 and 12 months and one 28 months after stopping treatment.

In a study ${ }^{2}$ on 34 relapses of PB leprosy, the mean incubation time for relapse was 2.73 years and the median 2.7 years, with a $95 \%$ confidence limit between 1 and 2 years and 3 years; $55 \%$ of relapses occurred within the first 3 years of follow-up. The confidence intervals determined in our study are of the sa me order of magnitude, although somewhat larger, this might be due to the smaller sample size of our study group.

In a study of 105 relapses $^{3}$ in PB leprosy patients followed during $3 \frac{1}{2}$ years, note that $75 \%$ of them were diagnosed within the first 2 years after stopping treatment.

Another study ${ }^{4}$ was also aimed at defining the optimalduration of therapy but the information necessary for the present purpose can be derived from their figures. In this study, $48 \%$ of relapses after stopping treatment occurred during the first 2 years, the $95 \%$ confidence limit of this figure being between 0 and $4 \cdot 1$ years.

Differences between the studies mentioned are the duration of follow-up and the diagnostic criteria. Follow-up was shorter in two studies, average 22 months, ${ }^{1}$ and $3 \frac{1}{2}$ years, ${ }^{3}$ than in the other studies. Depending on the shape of the curve, a greater fraction of the total number of relapses may appear earlier after discontinuation of treatment, as was the case in the two above-mentioned studies.

Diagnostic criteria may also differ between clinicians, particularly for 
paucibacillary leprosy, where the criteria for 'activity' are rather ill defined and are somewhat subjective. However, in each study mentioned the same clinicians were responsible for the diagnosis of relapse and in the present study the more objective histopathological criterion was used. Furthermore, the frequency at which patients are seen or may be seen, particularly in ambulatory services, may also determine the moment at which a relapse is diagnosed or may possibly be diagnosed.

But in conclusion it may be stated that during a follow-up period of 3 years after stopping treatment in PB leprosy, $50 \%$ of relapses should be observed.

One may wonder whether relapses observed shortly (within less than 1 year) after stopping a treatment of short duration are real relapses or upgrading reactions. Only careful future studies will perhaps be able to answer this question.

It is sometimes argued that late relapses occurring many years after stopping treatment may be the result of reinfections and thus are not necessarily relapses. This is certainly true but impossible to prove or disprove. Therefore, it is preferable for the calculation of the incidence of relapses to adopt the 'worst' hypothesis and consider them as relapses.

Another argument frequently raised during discussions is that all data presently available are from patients treated with dapsone monotherapy and that these figures could be entirely irrelevant when powerful bactericidal drugs such as rifampicin and/or ethionamide are used. This results from a confusion of two different issues. One is the antibacterial treatment, aimed at the killing of the etiological miroorganism, the other is the time necessary for viable microorganisms, subsisting after the end of the antibacterial treatment, to multiply again to such a level that new clinico-pathological lesions appear. The minimal duration of the antibacterial treatment necessary to kill all responsible microorganisms will certainly differ for drugs with differing bactericidal activity. However, the incubation time for relapses is a function of the presence of viable organisms present at the end of the antibacterial treatment, their number and the immunological defence capability of the host. However, the generation time of the bacilli is unaffected by the drugs previously administered.

Thus a clear distinction should be made between the incidence of relapse and the kind and duration of antibacterial therapy. The latter will certainly influence the incidence of relapses but not their incubation time. This is so in all infectious diseases in general and in tuberculosis in particular: the incubation time for relapses is not influenced by the drugs previously administered. The main difference between leprosy and tuberculosis in relation to the incubation time of relapses is that in the latter $90 \%$ appear within the first year after stopping treatment, whereas in leprosy only $50 \%$ are diagnosed within the first 3 years. This is most probably related to the widely different generation times of the two etiological agents. After stopping treatment one viable Mycobacterium tuberculosis needs about a month to grow out to a population of $10,{ }^{7}$ while one viable Mycobacterium leprae needs 1 year. The possibly greater natural immunity of 
humans against $M$. leprae may, furthermore, delay the appearance of clinical disease in this infection.

And the same rules also hold for multibacillary leprosy: relapse being a function of the existence of viable organisms at the end of treatment, their unaltered generation time in the absence of antileprosy drugs and the lower antileprosy immunity of the patient as compared with paucibacillary leprosy, $50 \%$ of relapses in multibacillary leprosy should also appear within 3 years af ter stopping treatment. We hope that future studies will clarify this point.

\section{Acknowledgments}

This study was made while the Leprosy Laboratory of the Institute of Tropical Diseases, Antwerp was financed by the Damien Foundation, Brussels, Nationaal Fonds voor Wetenschappelijk Onderzoek, Brussel and the Chemotherapy of Leprosy (Thelep) component of the UNDP/World Bank/WHO Special Programme for Research and Training in Tropical Diseases. A. De Muynck performed the statistical calculations.

\section{References}

1 Lowe J. The late results of sulphone treatment of leprosy in East Nigeria. Lepr Rev, 1954; 25: 113-24.

2 Ekambaram V. Duration of treatment for 'Disease Arrest' of non-lepromatous cases and relapse rate in these patients. Lepr Rev, 1979; 50: 297-302.

3 Touw-Langendijk EMJ, Naafs B. Relapses in leprosy after release from control. Lepr Rev, 1979; 50: $123-7$.

${ }^{4}$ Vellut C, Lechat MF, Misson CB. Tuberculoid relapses in leprosy. In Proc XI Int Lepr Congress. Latapi F, Sand A, Rodriguez O, Malacara A, Browne SG (eds), Amsterdam-Oxford-Princeton: Excerpta Medica, 1980. 